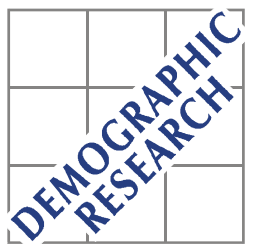

Demographic Research a free, expedited, online journal of peer-reviewed research and commentary in the population sciences published by the Max Planck Institute for Demographic Research Doberaner Strasse 114 - D-18057 Rostock · GERMANY www.demographic-research.org

DEMOGRAPHIC RESEARCH

VOLUME 6, ARTICLE 13, PAGES 355-382

PUBLISHED 23 MAY 2002

www.demographic-research.org/Volumes/Vol6/13/

DOI: 10.4054/DemRes.2002.6.13

\title{
To Tie the Knot or Not:
}

Cohabitation, Marriage and Individuals' Attitudes to Risk

Padma Rao Sahib

Xinhua Gu

(C) 2002 Max-Planck-Gesellschaft. 


\section{Table of Contents}

1 Introduction 356

2 Search in the marriage market 357

2.1 The modelling framework 357

$\begin{array}{lll}2.2 & \text { The model with cohabitation } & 359\end{array}$

3 The decision making process 362

3.1 Stage 1: being single 362

3.2 Stage 2: should we live together? 363

3.3 Stage 3: should we marry? 363

3.4 Derivation of optimal strategies 364

$4 \quad$ The class partition 366

5 Risk premia, cohabitation and marriage $\quad 370$

6 Conclusion 374

$\begin{array}{ll}\text { Acknowledgements } & 374\end{array}$

$\begin{array}{ll}\text { Appendices } & 375\end{array}$

$\begin{array}{ll}\text { Notes } & 379\end{array}$

References 380 


\title{
To Tie the Knot or Not: Cohabitation, Marriage and Individuals' Attitudes to Risk
}

\author{
Padma Rao Sahib ${ }^{1}$ \\ Xinhua $\mathbf{G u}^{2}$
}

\begin{abstract}
This paper introduces imperfect information, learning, and risk aversion in a two sided matching model. The model provides a theoretical framework for the commonly occurring phenomenon of cohabitation followed by marriage, and is consistent with empirical findings on these institutions. The paper has three major results. First, individuals set higher standards for marriage than for cohabitation. When the true worth of a cohabiting partner is revealed, some cohabiting unions are converted into marriage while others are not. Second, individuals cohabit within classes. Third, the premium that compensates individuals for the higher risk involved in marriage over a cohabiting partnership is derived. This premium can be decomposed into two parts. The first part is a function of the individual's level of risk aversion, while the second part is a function of the difference in risk between marriage and cohabitation.
\end{abstract}

\footnotetext{
${ }^{1}$ Corresponding author, Department of International Economics and Business, Faculty of Economics, University of Groningen, PO Box 800, 9700 AV Groningen, The Netherlands, phone +31 50363 3724, fax +31 50 363 7337, email: p.rao.sahib@eco.rug.nl

${ }^{2}$ Department of Economics, University of Toronto, Toronto, Canada
} 


\section{Introduction}

There are not many among the married who can claim that their spouses have remained the same as they were on their wedding day. Individuals marry and subsequently discover new characteristics in their spouses. Some of these characteristics may come as pleasant surprises; finding out that your spouse is an excellent cook is one example. However, it is evident from the high rate of divorce in many countries that many surprises are not as pleasant.

Because divorce can be psychologically painful and costly, some researchers hypothesize that couples may choose to cohabit instead of marrying. Whether cohabitation is a result of increased divorce rates is unclear. Waters and Ressler (1999) study state level data on cohabitation and divorce and conclude that the causality between divorce and cohabitation runs in both directions. What is clear, however, is that cohabitation as a life style has gained in popularity in recent decades. This is particularly the case in North America and Europe. For example, between the mid 1970s and the late 1980s the percentage of women aged 20-24 who were in cohabiting unions rose from $11 \%$ to $49 \%$ in France (Kiernan 1996).

Although prevalent, cohabitation is short-lived. It tends to precede marriage rather than replace it. Bumpass and Sweet (1989) use data from the United States and find that $40 \%$ of all cohabiting couples either marry or stop living together within a year, and only a third of cohabiting couples are still cohabiting after two years. They also find that $60 \%$ of those who marry after living in a cohabiting union, marry their cohabiting partners. This is corroborated by more recent data from the United Kingdom. Ermisch and Franscesconi (2000) also find that more than half of first cohabiting unions are converted into marriage. The most frequently cited reason for unmarried individuals living together is to assess compatibility before marriage (Bumpass, Sweet and Cherlin 1991). This indicates that cohabitation is a probationary period for many couples.

The goal of this paper is to develop a theoretical model consistent with the observed facts on cohabitation and marriage. It is evident from empirical findings that cohabitation is often transitory and is sometimes viewed as a kind of trial marriage. It appears to involve less commitment as cohabiting unions are more likely to dissolve than marriages.

The model developed in this paper adopts the view that couples cohabit because they want to evaluate one another as potential spouses. They find out each others' suitability as spouses while living together. Upon finding this out, they either marry or separate. In addition, this study incorporates imperfect information, learning and risk aversion into a two-sided search-matching model. These are new features in the growing literature on such models (see Burdett and Coles 1999 for a survey). It extends Rao Sahib and Gu (2000), in which premarital cohabitation and subsequent marriage are investigated in a risk neutral setting. 
The remainder of the paper is organized as follows. Section 2 discusses the model. Section 3 describes the decision making of couples. Section 4 shows that cohabiting unions occur within classes. The role of risk in cohabiting and marital unions is discussed in section 5. Section 6 concludes the paper.

\section{Search in the marriage market}

\subsection{The modelling framework}

The framework used in this paper is a steady state matching model with nontransferable utility and heterogeneous agents as developed in Burdett and Coles (1997), hereafter simply BC.

A model of a decentralized marriage market in which positive assortative mating arises as an equilibrium outcome is developed in $\mathrm{BC}$. The positive assortative mating found in $\mathrm{BC}$ is in terms of a comprehensive index. This index, termed pizazz, captures the worth of an individual as a marriage partner. If a couple marry, they each receive as utility the pizazz of the other. Equilibrium is characterized by a class partition. Men and women form classes with marriages occurring only among members of the same class. There exists an ordering among classes. Men and women with high pizazz marry one another while men and women with low pizazz marry one another. Not all two-sided search models yield positive assortative mating as an equilibrium outcome; see for example, Shimer and Smith (2000).

Narcissism is ruled out in the BC model. Having high pizazz is useful only because it allows one to attract opposite sex individuals who also have high pizazz, and increases the utility from marriage. The optimal policy for a single individual to follow is to marry the first single encountered of the opposite sex whose pizazz is above a certain reservation level. The steady state is characterized by a constant distribution of pizazz among the pool of single individuals and a balanced flow of market exits and entries.

In this paper, we deviate from the BC framework in four ways. First, BC assume that an individual's pizazz can be instantly observed on contact. In contrast, we assume that single individuals can observe the true pizazz of a potential partner only imperfectly (we retain the term pizazz to mean the worth of an individual as a marriage partner). This provides a motivation for cohabiting unions which are initiated by single men and women in an attempt to learn each other's true worth as marriage partners. Cohabitation is of course just one interpretation of this information collecting period. It can also be called courtship or dating. Couples continue to cohabit until true pizazz is revealed, and at that point, they either marry or separate. Our approach is similar to Chade (1999), in which individuals also observe one another's type imperfectly. However, in Chade's 
model individuals never learn one others' true type. They leave the market forever after matching on imperfectly observed type.

Second, in BC, individuals leave the marriage market forever through marriage or death, and there is an exogenous in-flow of new singles into the market. In our model, cohabiting unions sometimes dissolve and individuals re-enter the marriage market. This is the outcome of the revealed true type of one partner being too low for the other.

Third, it is assumed in $\mathrm{BC}$ that individuals are risk neutral. We relax the assumption of risk neutrality and consider the case when individuals are risk averse. This allows us to derive a general expression for the risk premium for marriage over a cohabiting union.

Fourth, although it is not explicitly stated in $\mathrm{BC}$, the distribution of offers of marriage from opposite-sex singles faced by an agent first-order stochastically dominates the distribution faced by an agent from a lower pizazz class. This continues to hold for the offer distributions in our model. However, the introduction of uncertainty and learning allows us to use the concept of second-order stochastic dominance to analyze the role of risk in cohabiting and marital unions.

We retain, however, several of the assumptions made in $\mathrm{BC}$. We assume that a large and equal number of male and female singles participate in a marriage market. Clearly, as men and women either cohabit or marry and leave the singles market, the distribution of pizazz will change over time. However, we assume, as in BC, that singles are only partially rational and believe that the environment is stationary. This is an important assumption because we assume that individuals are believed to follow stationary strategies.

Individuals are assumed to adopt utility maximizing strategies given the behavior of other singles in the market. In particular, it is assumed that participants in the marriage market seek to maximize the expected discounted lifetime utility by searching for the best possible matches, discounted at a rate $r$ to the present. The optimal policy to be followed by individuals consists of a certain set of opposite sex singles to whom they will make offers of cohabitation if they meet.

The market distributions of pizazz for men and women which are consistent with flowin and flow-out distributions that are equal, make up the set of steady state equilibria. Our focus, however, is on the decision problem faced by individuals' Nash equilibrium rather than the market equilibria. In our model, the calculation of the steady state is complicated by the fact that in addition to outflows of singles due to marriage, cohabiting unions may dissolve resulting in individuals re-entering the singles market. Therefore for simplicity, death and divorce are omitted in this paper.

We next describe the model in which couples are assumed to first cohabit and then marry. 


\subsection{The model with cohabitation}

In the rest of the paper, we simplify exposition by considering the decision maker to be a single woman who follows an optimal search strategy to find a potential marriage partner (the arguments are identical for the case of a single man, and are dealt with by symmetry). We assume that when a woman meets a man, she only observes his pizazz imperfectly. She observes $y$, though his true pizazz is $x$. Symmetrically, the man also obtains a noisy observation on the woman's pizazz. We assume that singles believe that the distributions of both true pizazz $x$ and observed pizazz $y$ are time-invariant.

The distributions of true pizazz among men and women are denoted $G_{M}(x)$ and $G_{W}(x)$ respectively. For simplicity, we assume $G_{M}(x)=G_{W}(x)=G(x)=P(X \leq x)$ with support $[\underline{x}, \bar{x}]$. In this sense, the search-matching process is random.

We assume that observed pizazz is the sum of true pizazz and random noise. That is, the woman observes the man's $y$, a realization of $Y$, the random variable used to denote observed pizazz. We assume that $Y=X+\varepsilon$ and $\varepsilon$ is independent of $X$ (denoted $\varepsilon \perp X$ ). Also, it is assumed that $E(\varepsilon)=0$ and $\operatorname{Var}(\varepsilon)$ is small.

Upon observing $y$, the woman forms an expectation of the true pizazz of the man, $E(X \mid Y=y)$ denoted $m(y)$ or simply $m$. Although $m$ is expected true pizazz conditional on observed pizazz, for the sake of brevity, we will refer to $m$ simply as expected pizazz.

Here, $m$ can be interpreted is the Bayes estimate of $x$, denoted $\widehat{x}$. This estimate is obtained by minimizing a risk function: $\min _{\widehat{x}} E_{x \mid y} \frac{1}{2}(\widehat{x}-x)^{2}$. Notice that this is the expectation of a quadratic loss function with respect to a posterior distribution $Q_{X \mid Y}(x \mid y)$, as defined later. For example, consider the case when $X \sim N\left(\mu, \sigma^{2}\right), \varepsilon \sim N\left(0, \sigma_{\varepsilon}^{2}\right)$ and $Y=X+\varepsilon$ with $\varepsilon \perp X$. Then, $\widehat{x}=m(y)=w \mu+(1-w) y$ where $w=\sigma_{\varepsilon}^{2} /\left(\sigma^{2}+\sigma_{\varepsilon}^{2}\right)$.

We make two assumptions. The first (A1) is that $m^{\prime}(y)>0$. This implies that $m$ is increasing in $y$. This captures the common view that first impressions matter. This assumption is satisfied in the normal distribution case mentioned above, as $m^{\prime}(y)=1-$ $w>0$.

Moreover, the assumption that $m$ is increasing in $y$ is useful because $m$ is then invertible. The support of $M$ denoted $[\underline{m}, \bar{m}]=[m(\underline{y}), m(\bar{y})]$. Denoting the distribution of $M$ by $F(m)$ we can write $F(m)=F_{Y}(y(m))$.

For this woman, decisions regarding whether or not to enter a cohabiting relationship are based on $m$. Notice that $m$ more informative than $y$ since it contains inference about $x$ from $y$. Also, it enables the woman to increase the precision of her inference about the man's true pizazz. For the case of the normal distribution mentioned above, $M \sim$ $N\left(\mu, \sigma_{m}^{2}\right)$ where $\sigma_{m}^{2}=\sigma^{4} /\left(\sigma^{2}+\sigma_{\varepsilon}^{2}\right)$. In this case, $\sigma_{m}^{2}<\sigma^{2}<\sigma_{y}^{2}$, where $\sigma_{y}^{2}=$ $\sigma^{2}+\sigma_{\varepsilon}^{2}$. Note that in estimating a particular $x$, expected pizazz $m$ is more precise than 
observed pizazz $y$ since $\sigma_{m \mid x}^{2}\left(=(1-w)^{2} \sigma_{\varepsilon}^{2}\right)$ is less than $\sigma_{y \mid x}^{2}\left(=\sigma_{\varepsilon}^{2}\right)$.

The second assumption (A2) we make involves the distribution of the man's true pizazz conditional on his observed pizazz given he has cohabited with the woman denoted $Q_{X \mid Y}(x \mid y)=P(X \leq x \mid Y=y)$. By definition, the mean of this distribution is $m$ (see the Appendix (1)).

Using $y=y(m)$, the conditional distribution $Q_{X \mid Y}(x \mid y(m))$ can be expressed as $Q(x, m)$. We assume in $\mathrm{A} 2$ that $\partial Q(x, m) / \partial m<0$. This assumption implies that for any given $m_{B}<m_{A}$ we see that $Q\left(x, m_{A}\right)<Q\left(x, m_{B}\right)$ for all $x \in S_{X}$. That is, $Q\left(x, m_{A}\right)$ first order stochastically dominates $Q\left(x, m_{B}\right)$. Therefore, $E_{Q\left(x, m_{B}\right)} J(x)$ $<E_{Q\left(x, m_{A}\right)} J(x)$ for a non-decreasing function $J(x)$.

For example, suppose that a single woman encounters two single men, denoted $A$ and $B$ with observed pizazz $y_{A}$ and $y_{B}$ respectively. Assume that man $A$ appears to be the better partner than man $B$. That is, $y_{B}<y_{A}$. From A1 it follows that $m^{\prime}(y)>0$, and therefore $m_{B}<m_{A}$. Then, A2 implies that the utility of the woman marrying man A would be higher than the utility obtained from marrying man $\mathrm{B}$.

This assumption holds for the normal distribution mentioned above. In this case, $\partial Q(x, m) / \partial m=-\frac{1}{\sigma_{1}} \phi\left(\frac{x-m}{\sigma_{1}}\right)<0$ where $\phi(\cdot)$ is the standard normal density. Recall that $(X \mid y) \sim N\left(m, \sigma_{1}^{2}\right)$ where $\sigma_{1}^{2}=w \sigma^{2}$.

In addition, a parameterizing variable is needed to take into account the effect of the woman's own attractiveness on opportunities available to her in the marriage market (we still treat her as the decision maker, using tilde to represent her characteristics). A woman with high pizazz is able to attract men who also have high pizazz. However, the woman may have a 'bad' day, and appear less attractive than she really is. That is, her observed pizazz $\widetilde{y}$ is the sum of $\widetilde{x}$ and a large negative draw $\widetilde{\varepsilon}$ from the noise distribution. Alternatively, she may be lucky and appear more attractive than she really is, in which case the noise drawn is positive and large. Given that $\widetilde{y}$ is an imperfect indicator of true pizazz, one could ask if a possible strategy may be for the woman to simply state her true pizazz, when she meets a man. However, this is not credible. Men who encounter a woman are more likely to trust their own judgment than the pizazz stated by the woman. They believe in their own estimate $\widetilde{m}$ of her true pizazz $\widetilde{x}$ based on observed pizazz $\widetilde{y}$.

Notice, however, that a woman's $\widetilde{m}$ is still random from her own point of view. She does not know the future realization of the noisy version of her true pizazz observed by men. However, the expected discounted utility of receiving no offers of cohabitation and continuing to be single, denoted $R$, has to be non-stochastic in search models. Therefore, as $\widetilde{m}$ is stochastic, we parameterize $R$ by $\widetilde{m}_{e}$ instead of $\widetilde{m}$. Here, $\widetilde{m}_{e}$ is the woman's own expectation of her $\widetilde{m}$ conditional on her private knowledge of her true pizazz $\widetilde{x}$, whereas $\widetilde{m}$ is men's expectation of her $\widetilde{x}$ based on her $\widetilde{y}$. We refer to this woman (the decision maker) as an $\widetilde{m}_{e}$-type woman. That is, $\widetilde{m}_{e}=E(m(\widetilde{y}) \mid \widetilde{x})=E_{\varepsilon}(m(\widetilde{x}+\widetilde{\varepsilon}))$ 
$=m_{e}(\widetilde{x})$. In the case of the normal distribution, $\widetilde{m}_{e}=w \mu+(1-w) \widetilde{x}$. Notice that this is a weighted average of two quantities. The first is her true pizazz, $\widetilde{x}$. The second quantity is her estimate of the estimate made by the men she meets, which is $\mu$. Note that $E(\widehat{x})=E(M)=E(E(X \mid Y))=E(X)=\mu$, which also holds after adding the tilde symbol.

There exists a set of men who are willing to cohabit with a $\widetilde{m}_{e}$-type woman. We denote the distribution of $m$ among such men by $F\left(m \mid \widetilde{m}_{e}\right)$, with $\widetilde{m}_{e}$ being the parameterizing variable which reflects the woman's opportunities in the market.

We assume, as it is often the case, that singles have difficulty contacting members of the opposite sex and meet bilaterally (this is in contrast to Bloch and Ryder 2000 in which couples are matched via a centralized matching procedure). To capture this uncertainty in initiating a cohabiting relationship, we assume that for any single in the market, the rate at which offers of cohabitation arrive follows a Poisson process. We denote by $\alpha$ the total arrival rate of offers for cohabitation from all men (or women) faced by a particular woman (or man). As men and women face different opportunities because of differences in their own attractiveness as a decision-maker, only a fraction of these offers, denoted $\alpha\left(\widetilde{m}_{e}\right)$, are received by a woman with $\widetilde{m}_{e}$, and $\alpha\left(\widetilde{m}_{e}\right) \leq \alpha$.

As cohabiting unions are initiated based on a noisy signal of an individual's pizazz, some cohabiting unions progress to marriage when couples discover each other's true worth to be high enough as marriage partners while others dissolve. We assume that cohabiting couples also have difficulty discovering each others' true pizazz when deciding whether the partnership can evolve into marriage. This uncertainty is also characterized by a Poisson process with parameter $\lambda$, the total arrival rate of new signals that lead to the revelation of the partner's true type. If no such signals arrive, couples stay in their current cohabiting unions. $\lambda$ can also be viewed as the arrival rate of marriage offers faced by either sex during cohabitation. The transition to marriage is not random, but depends on whether the partner's revealed true pizazz is high enough.

We can consider the matching process leading to marriage as occurring in three stages. In the first stage, which can be regarded as a "pre-draw" stage, a single individual is in the marriage market waiting for offers that arrive randomly. In this stage, she only knows the distribution of $M, F(m)$. Since $M=m(Y)=E(X \mid Y)$, the source of the randomness of $M$ is $Y$, which has yet to be drawn.

In the second stage, the woman meets a man. This implies that she draws $x$ but observes $y$, and calculates $m$. She chooses to cohabit with such a $m$-type man if his $m$ exceeds a certain threshold level, denoted $m_{r}$. This threshold is her reservation demand for the man's expected true pizazz conditional on his observed pizazz. If not, she remains single and the process repeats itself in the next period. It is of course possible that she herself is rejected by the man she has encountered in which case she has no choice but to remain single until the next period. 
The revelation of true pizazz is stochastic, and marks the third stage. We assume, for simplicity, that learning occurs only once and only during cohabitation. At this point, each party must decide whether to maintain or quit the current match. If the couple choose to

maintain the match, they are assumed to establish a formal marriage and leave the market forever. If the cohabiting couple choose not to marry, they are assumed to separate and return to the singles pool.

This artificial distinction between stages is made only to aid understanding. Events, in fact, occur at random intervals in the context of the Poisson approximation that underlies the processes of cohabitation and marriage in the continuous-time setting. The next section develops the optimal policy to be followed at each stage. For ease of exposition, we continue to assume that the decision maker is a woman.

\section{The decision making process}

\subsection{Stage 1: being single}

This is the "pre-draw" stage, when a $\widetilde{m}_{e}$-type single woman awaits offers of cohabitation. At this stage, she knows neither $x$ nor $y$ before any offer arrives. Her knowledge of the market is only $F(m)$. Standard dynamic programming arguments yield,

$$
R\left(\widetilde{m}_{e}\right)=\frac{1}{1+r h}\left[u_{\widetilde{m}_{e}}(0) h+\alpha\left(\widetilde{m}_{e}\right) h E_{m} V_{\widetilde{m}_{e}}(m)+\left(1-\alpha\left(\widetilde{m}_{e}\right) h\right) R\left(\widetilde{m}_{e}\right)\right]+o(h) .
$$

In the above, $R\left(\widetilde{m}_{e}\right)$ is the expected discounted utility of the $\widetilde{m}_{e}$-type woman being single in the current period and following an optimal policy from the next period onward. The term $u_{\tilde{m}_{e}}(0)=\left.u_{\tilde{m}_{e}}(m)\right|_{m=0}$ is the utility she receives in the current period of short length $h$ when no man has been encountered. The term $\alpha\left(\widetilde{m}_{e}\right) h$ is the probability that the woman will receive an offer of cohabitation during a future time interval $h . E_{m^{\prime}} V_{\widetilde{m}_{e}}\left(m^{\prime}\right)$ is the expected discounted value of having a future offer from an $m^{\prime}$-type man in that interval and behaving optimally later on. The probability that no offer of cohabitation will be received in the time interval $h$ is $1-\alpha\left(\widetilde{m}_{e}\right) h$ in which case the woman has no choice but to remain single.

Since narcissism is ruled out, we have $u_{\tilde{m}_{e}}(m)=u(m)$. Also, we assume for now that $u(m)=m$. Note that $o(h) / h \rightarrow 0$ as $h \rightarrow 0$. We can rewrite (1) to obtain

$$
R\left(\widetilde{m}_{e}\right)=\frac{\alpha\left(\widetilde{m}_{e}\right)}{r+\alpha\left(\widetilde{m}_{e}\right)} E_{m} V_{\widetilde{m}_{e}}(m) .
$$

No decision can be made at this stage, and only the Poisson process with parameter $\alpha$ determines a random outcome. 


\subsection{Stage 2: should we live together?}

At this stage, the $\widetilde{m}_{e}$-type woman encounters a man and based on his observed pizazz, estimates his true pizazz. That is, she calculates $E(X \mid Y=y)$ or $m$. The woman is confronted with two choices: continue to be single and receive $R\left(\widetilde{m}_{e}\right)$ or enter into a cohabiting union with the man she has encountered. The value function at this stage is:

$$
V_{\widetilde{m}_{e}}(m)=\max \left\{\varphi_{\widetilde{m}_{e}}(m), R\left(\widetilde{m}_{e}\right)\right\} .
$$

In the above expression, $\varphi_{\widetilde{m}_{e}}(m)$ is the expected discounted utility of the $\widetilde{m}_{e}$-type woman accepting an offer of cohabitation from an $m$-type man in this period and behaving optimally from the next period onwards. This term can be written as follows:

$$
\varphi_{\widetilde{m}_{e}}(m)=\frac{1}{1+r h}\left[u(m) h+\lambda h E_{x \mid m} J_{\widetilde{m}_{e}}(x)+(1-\lambda h) \varphi_{\widetilde{m}_{e}}(m)\right]+o(h) .
$$

The first term $u(m) h$ is the utility received in the current short time interval $h$ of being in a cohabiting union with the $m$-type man. The term $\lambda h$ is the probability in the next time interval $h$ of the woman receiving a new signal which leads to the revelation of the cohabiting partner's true pizazz $x$. The value to the woman of receiving an offer for marriage from that partner during this future interval and behaving optimally later on is $J_{\widetilde{m}_{e}}(x)$. In the event that no offer of marriage is received (with $x$ not revealed) during the next time interval $h$, and this occurs with probability $(1-\lambda h)$, the woman will remain in the current cohabiting relationship. Then, as $h \rightarrow 0$ (3) reduces to

$$
\varphi_{\widetilde{m}_{e}}(m)=\frac{m+\lambda E_{x \mid m} J_{\widetilde{m}_{e}}(x)}{r+\lambda} .
$$

It should be pointed out that although there is no uncertainty once true pizazz is revealed at some moment during cohabitation, the revelation of true pizazz is a random event. It is only after this occurs that the couple can decide whether to marry or separate. However, the optimal policy to be followed is determined ex-ante, before any offers of marriage are made. Therefore, we need to introduce the Poisson parameter $\lambda$, which links the cohabitation and marriage stages of the model. The optimal policy for stage 2 is derived in section 3.4.

\subsection{Stage 3: should we marry?}

Stage 3 is when the woman finds out the true pizazz $x$, of her cohabiting partner. She then chooses to either separate from him or marry him. We assume that the woman receives 
as utility, the true pizazz of her husband in each time period following marriage. That is, we assume that $u_{\widetilde{m}_{e}}(x)=x$. It follows then that the lifetime utility from marriage is $\psi_{\widetilde{m}_{e}}(x)=x / r=\psi(x)$. This is based on the assumption that once a couple marry, they stay married forever and never return to the singles market. If the woman does not marry, she returns to the singles market and the expected discounted utility she receives is then $R\left(\widetilde{m}_{e}\right)$, as discussed in stage 1 . Therefore, the value function at this stage can be written as

$$
J_{\widetilde{m}_{e}}(x)=\max \left\{\psi_{\widetilde{m}_{e}}(x), R\left(\widetilde{m}_{e}\right)\right\}
$$

The optimal strategy to be followed at this stage is a reservation pizazz policy. The woman marries her cohabiting partner if his true pizazz, $x \geq x_{r}$ where $x_{r}=r R\left(\widetilde{m}_{e}\right)$ $=\psi^{-1}\left(R\left(\widetilde{m}_{e}\right)\right)$. In this expression, $x_{r}$ is the minimum pizazz level of a man as the marriage partner acceptable to the $\widetilde{m}_{e}$-type woman. This result depends on the constancy of $R\left(\widetilde{m}_{e}\right)$ w.r.t. $x$, which is shown next.

\subsection{Derivation of optimal strategies.}

To show that the optimal policy to be followed in stage 2 is a reservation pizazz strategy, we must demonstrate that $\varphi_{\widetilde{m}_{e}}(m)$ is strictly increasing in $m$. To this end, we first examine the expectation of value function in stage 3 (see Appendix (2) for details):

$$
E_{x \mid m} J_{\widetilde{m}_{e}}(x)=\frac{1}{r} \int_{\psi^{-1}\left(R\left(\widetilde{m}_{e}\right)\right)}^{\bar{x}}[1-Q(x, m)] d x+R\left(\widetilde{m}_{e}\right)
$$

which is a function of $\left(m, \widetilde{m}_{e}\right)$.

This allows for an interpretation of A2. Notice that $J_{\widetilde{m}_{e}}(x)$ is non-decreasing in $x$. Using $E_{Q}(\cdot)$ to denote the expectation taken w.r.t. $x$ under distribution $Q$, we see that for $m_{A}>m_{B}$,

$$
E_{Q\left(x, m_{A}\right)} J_{\widetilde{m}_{e}}(x)>E_{Q\left(x, m_{B}\right)} J_{\widetilde{m}_{e}}(x)
$$

since $Q\left(x, m_{A}\right)$ first-order stochastically dominates $Q\left(x, m_{B}\right)$. This inequality can clearly be seen to hold by examining (5) under A2. This implies that when the woman cohabits with a $m_{A}$-man as opposed to a $m_{B}$-man, she will obtain a higher level of the expected discounted utility from following an optimal strategy in seeking a marriage partner.

Assumption A2 is also important in terms of the mathematics involved in proving that 
$\varphi_{\widetilde{m}_{e}}(m)$ is increasing in $m$. Differentiating (5) w.r.t. $m$ we obtain,

$$
\frac{\partial E_{x \mid m} J_{\tilde{m}_{e}}(x)}{\partial m}=\frac{1}{r} \int_{\psi^{-1}\left(R\left(\tilde{m}_{e}\right)\right)}^{\bar{x}}\left[-\frac{\partial Q(x, m)}{\partial m}\right] d x>0,
$$

because of A2. Then differentiating (4) w.r.t. $m$ yields:

$$
\frac{\partial \varphi_{\tilde{m}_{e}}(m)}{\partial m}=\frac{1}{r+\lambda}\left[1+\lambda \frac{\partial E_{x \mid m} J_{\widetilde{m}_{e}}(x)}{\partial m}\right]>0 .
$$

This ensures that a unique solution to the stage- 2 problem exists. The optimal strategy to be followed in stage 2 is also a reservation pizazz policy. An $\widetilde{m}_{e}$-type woman cohabits with a man if his estimated pizazz $m \geq m_{r}$ where $m_{r}=\varphi_{\tilde{m}_{e}}^{-1}\left(R\left(\widetilde{m}_{e}\right)\right)$.

We solve for the utility of being single in stage 1 for a $\widetilde{m}_{e}$-type woman as follows (see Appendix (3) for its derivation):

$$
\begin{aligned}
& R\left(\widetilde{m}_{e}\right)=\frac{\alpha\left(\widetilde{m}_{e}\right)}{r(r+\lambda)} \int_{\substack{\varphi \sim_{m}^{-1} \\
\tilde{m}_{e}}}^{\bar{m}\left(R\left(\widetilde{m}_{e}\right)\right)}\left[1-F\left(m \mid \widetilde{m}_{e}\right)\right] \\
& \times\left[1-\frac{\lambda}{r} \int_{\psi^{-1}\left(R\left(\tilde{m}_{e}\right)\right)}^{\bar{x}} \frac{\partial Q(x, m)}{\partial m} d x\right] d m
\end{aligned}
$$

Notice that from the above equation, it is clear that $R\left(\widetilde{m}_{e}\right)$, which is only a function of $\widetilde{x}$, does not depend on particular values of $x$ or $m$. This constancy w.r.t. $m$ and $x$ is important in establishing that a reservation strategy is optimal in stages 2 and 3.

Blackwell's theorem (see Sargent 1987) ensures that there should exist a unique solution to this functional equation in $R\left(\widetilde{m}_{e}\right)$ which is continuous in $\left[\varphi_{\widetilde{m}_{e}}^{-1}\left(R\left(\widetilde{m}_{e}\right)\right), \bar{m}\right]$. By symmetry, we have another functional equation in $R\left(m_{e}\right)$ for an $m_{e}$-type man as the decision-maker, which is identical to (8) except that $m_{e}$ and $\widetilde{m}_{e}$ are exchanged. The assumption that men and women face the same underlying distributions, that is $G_{M}(x)$ $=G_{W}(x)$, allows us to use the symmetry argument. These two equations are used to derive the results on class partitioning which will be discussed in the next section.

Since the rest of the paper deals with matching and class partition, we simply denote the type of the decision maker as $\widetilde{m}_{e}$. The type of the potential partners of this decision 
maker is denoted $m_{e}$. As long as a sorting result is attained for one sex, the same can be derived for the other sex by symmetry. This symmetric equilibrium induces sorting of individuals at the aggregate level.

\section{The class partition}

In this section, we show that cohabiting unions occur only between couples in the same class and that this result holds for marriages as well. The number of classes is finite in both the cohabiting and the marital case. To prove these results, we establish a series of lemmas:

Lemma 1 During the search for a cohabiting partner, the distribution of $m$ among oppositesex singles faced by a $\widetilde{m}_{e}$-type single first-order stochastically dominates that faced by a lower $\widetilde{\widetilde{m}}_{e}$-type single. That is, $F\left(m \mid \widetilde{m}_{e}\right)<F\left(m \mid \widetilde{\widetilde{m}}_{e}\right)$ for $\widetilde{\widetilde{m}}_{e}<\widetilde{m}_{e}$.

Proof: In what follows, $m^{\prime}$ denotes the upper bound of the parameterizing variable $\widetilde{m}_{e}$.

$$
\begin{aligned}
F\left(m \mid \widetilde{m}_{e}\right) & =P\left(M \leq m \mid \widetilde{M}_{e} \leq m^{\prime}\right)=P\left(M \leq m \mid M \leq m^{\prime}\right) \\
& =F(m) / F\left(m^{\prime}\right), \quad \text { for } \underline{m} \leq m \leq m^{\prime}, \text { and } \widetilde{m}_{e} \leq m^{\prime}
\end{aligned}
$$

In the above, we substitute $m^{\prime}$ in place of the conditioning variable $\widetilde{m}_{e} ; m^{\prime}$ will be determined later in the matching process. We can replace $\widetilde{M}_{e}$ by $M$ because we assume that the distribution of pizazz is the same for both men and women. Notice that $F\left(m \mid \widetilde{m}_{e}\right)$ is a truncated distribution with truncated support $\left[\underline{m}, m^{\prime}\right]$. As $\widetilde{m}_{e}$ decreases, its upper bound may or may not fall and the resultant support will shrink if the bound does fall, thus generating a series of truncated distributions. Because $F\left(m \mid \widetilde{m}_{e}\right)<F\left(m \mid \widetilde{\widetilde{m}}_{e}\right)$ for $\widetilde{\widetilde{m}}_{e} \leq m^{\prime \prime}, \widetilde{m}_{e} \leq m^{\prime}$ and $m^{\prime \prime}<m^{\prime}$, we have the result that $F\left(m \mid \widetilde{m}_{e}\right)$ first-order stochastically dominates $F\left(m \mid \widetilde{\widetilde{m}}_{e}\right)$.

Incidentally, $Q(x, m)$ needs no truncation since its mean $m$ is restricted within the upper bound of $\widetilde{m}_{e}$. This is because the marriage search model is nested in the cohabitation search model. This can be seen in (8) and will become clearer in lemmas 3, 4 and 5 that follow. Therefore, the support for $Q(x, m)$ does not shrink with class partition.

Lemma 2 A higher-type single of either sex receives a higher arrival rate of the offers for cohabitation than his or her competitors of lower types. 
Proof: $\alpha\left(\widetilde{m}_{e}\right)=\alpha F\left(m^{\prime}\right)$ for $\widetilde{m}_{e} \leq m^{\prime}$, which says that $\alpha\left(\widetilde{m}_{e}\right)$ is calculated by discounting the overall arrival rate of cohabitation offers $\alpha$ at a rate of $\left[1-F\left(m^{\prime}\right)\right]$, because this is the probability no opposite-sex singles with type $m$ greater than $m^{\prime}$ are willing to propose to a $\widetilde{m}_{e}$-type person for cohabitation.

Notice that these lemmas already contain some of the elements related to class partitioning. A single is better off proposing to opposite-sex individuals with types no greater than the upper bound of her or his own type. However, the lower bound of preferences of individuals is not yet clear. These lemmas are concerned only with the upper bound of compatible partners' pizazz levels. Further results based on reservation pizazz policies are needed to identify the lower bound of a pizazz interval within which couples can be matched.

Lemma 1 may be interpreted as defining the opportunity set for a single of type $\widetilde{m}_{e}$ (say a woman), $\left[\underline{m}, m^{\prime}\right] \equiv \mathbf{M}_{o p p}$. Any opposite-sex singles with $m$ higher than $m^{\prime}$ are unattainable to this woman. The reservation pizazz policy derived from her search model determines the acceptance set $\left[m_{r}, \bar{m}\right] \equiv \mathbf{M}_{a c c}$. Any opposite-sex singles below the lower bound $m_{r}$ are considered unacceptable to this woman. The intersection of the two sets, $\mathbf{M}_{\text {opp }} \cap \mathbf{M}_{a c c}$, identifies the set of opposite-sex partners that are both attainable and acceptable. This is a set of mutually agreeable pizazz types. Note that the reservation demand by opposite-sex singles determines the upper bound of this set while the single's own reservation demand decides its lower bound.

Lemma 3 The best individual of either sex accepts any member of the opposite sex also in the top-class. That is, the single whose $\widetilde{m}_{e}=\bar{m}$ will cohabit with a member of the opposite sex with $m \in\left(m_{1}, \bar{m}\right]$ and marry a member of the opposite sex with $x \in\left(x_{1}, \bar{x}\right]$.

Proof: Consider the case of the woman whose $\widetilde{m}_{e}=\bar{m}$, whom we refer to as the best woman. Using lemmas 1 and 2, we can show that $F(m \mid \bar{m})=F(m)$ for $m \in[\underline{m}, \bar{m}]$, and $\alpha(\bar{m})=\alpha$. From this and from (8), we find that the expected discounted utility of being single for the best woman satisfies the following equation:

$$
\bar{R} \equiv R(\bar{m})=\frac{\alpha}{r(r+\lambda)} \int_{\varphi \frac{-1}{m}(\bar{R})}^{\bar{m}}[1-F(m)]\left[1-\frac{\lambda}{r} \int_{\psi^{-1}(\bar{R})}^{\bar{x}} \frac{\partial Q(x, m)}{\partial m} d x\right] d m
$$

Solving this equation for $\bar{R}$ determines the best woman's reservation demand for the pizazz types of her cohabitants and spouses. We define $m_{r}(\bar{m})=\varphi_{\bar{m}}^{-1}(\bar{R}) \equiv m_{1}$ and $x_{r}(\bar{m})=\psi^{-1}(\bar{R}) \equiv x_{1}$.

We have now established that the best woman will cohabit with men whose $m \in$ $\left(m_{1}, \bar{m}\right]$ and marry men with $x \in\left(x_{1}, \bar{x}\right]$. We refer to men whose $m \in\left(m_{1}, \bar{m}\right]$ as 
making up the 'top-class' for cohabiting unions and men whose $x \in\left(x_{1}, \bar{x}\right]$ as making up the top-class for marital unions. By symmetry, the top-class of women can also be identified. Notice that the best man (or woman) will not necessarily cohabit with or marry the best woman (or man). This is the outcome of a positive discount rate and the difficulty in encountering singles of the opposite sex.

Lemma 4 A member of the top-class of either sex cohabits with and marries any member of the top-class of the opposite sex.

Proof: This is a natural corollary of lemma 3, and can be proved rigorously as follows. As we have shown that the best man will accept any woman in the top-class as a partner, all men must be willing to accept a woman of this class. This implies that any woman with $\widetilde{m}_{e} \in\left(m_{1}, \bar{m}\right)$ faces the same prospects as the best woman. She then faces the entire distribution of pizazz among men and the total arrival rate of offers from all men. Therefore, for such a woman, $F\left(m \mid \widetilde{m}_{e}\right)=F(m)$ for $m \in[\underline{m}, \bar{m}]$, and $\alpha\left(\widetilde{m}_{e}\right)=\alpha$. Substituting these quantities into (8) yields the expected discounted utility of being single for such a woman:

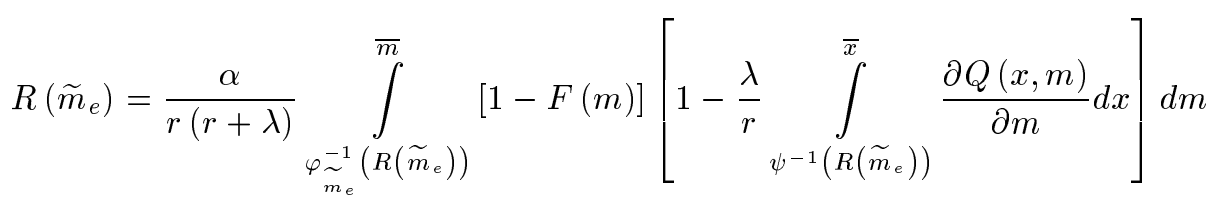

Equations (4) and (5) and lemma 2 imply $\varphi_{\widetilde{m}_{e}}^{-1}(\cdot)=\varphi_{\bar{m}}^{-1}(\cdot)$. Notice that (10) and (9) are identical except that the term $R(\bar{m})$ appears in (9) while the term $R\left(\widetilde{m}_{e}\right)$ appears in (10). Thus, $R\left(\widetilde{m}_{e}\right)=\bar{R}$ for $\widetilde{m}_{e} \in\left(m_{1}, \bar{m}\right]$. Therefore, the reservation demand for $m_{r}\left(\widetilde{m}_{e}\right)$ and $x_{r}\left(\widetilde{m}_{e}\right)$ by women in the top-class other than the best woman is identical to the reservation demand for $m_{1}$ and $x_{1}$ by the best woman.

Lemma 5 A member of the second-class of either sex cohabits with and marries any member of the second-class of the opposite sex. That is, singles with $m \in\left(m_{2}, m_{1}\right]$ cohabit with one another while couples whose $x \in\left(x_{2}, x_{1}\right]$ marry one another.

Proof: Consider a woman who is not in the top-class but she is the best among all women who are not in the top-class. That is, her $\widetilde{m}_{e}=m_{1}$. This woman will be rejected by all men in the top class, but she is the most attractive among women not in the top class. From lemmas 1 and 2 , she faces $F\left(m \mid m_{1}\right)=F(m) / F\left(m_{1}\right)$ for $m \in\left[\underline{m}, m_{1}\right]$, and $\alpha\left(m_{1}\right)=\alpha F\left(m_{1}\right)$. Using these results and applying (8) to the shrunk support $\left[\underline{m}, m_{1}\right]$ 
results in the expected discounted utility of being single for this woman:

$$
\begin{aligned}
R_{1} \equiv & R\left(m_{1}\right)=\frac{\alpha}{r\left(r+\lambda F\left(m_{1}\right)\right)} \int_{\varphi_{m_{1}\left(R_{1}\right)}^{m_{1}}}^{m_{1}}\left[F\left(m_{1}\right)-F(m)\right] \\
& \times\left[1-\frac{\lambda F\left(m_{1}\right)}{r} \int_{\psi^{-1}\left(R_{1}\right)}^{\bar{x}} \frac{\partial Q(x, m)}{\partial m} d x\right] d m
\end{aligned}
$$

Solving the above for $R_{1}$ identifies the men that form the second-class: $m \in\left(m_{2}, m_{1}\right], x$ $\in\left(x_{2}, x_{1}\right]$, where $m_{2}=\varphi_{m_{1}}^{-1}\left(R_{1}\right), x_{2}=\psi^{-1}\left(R_{1}\right)$. By symmetry, one can also obtain the second-class of women. By the same reasoning as in the proof of lemma 4, we have $R\left(\widetilde{m}_{e}\right)=R_{1}$ for $\widetilde{m}_{e} \in\left(m_{2}, m_{1}\right]$, and lemma 6 then follows.

Proposition 1 Singles (or cohabiting individuals) in class-i only accept opposite-sex singles (or cohabiting individuals) in the same class for cohabitation (or marriage), $1 \leq i \leq n$, where $n$ is a finite number.

Proof: Repeating the procedures in lemmas $3,4,5$, and applying mathematical induction, one can conclude that $R\left(\widetilde{m}_{e}\right)$ is a non-decreasing step function so that $R_{n-1}<\cdots<R_{1}$ $<\bar{R}$. Class partitioning is such that $(\underline{m}<) m_{n}<\cdots<m_{1}<\bar{m}$ and $(\underline{x}<) x_{n}<\cdots$ $<x_{1}<\bar{x}$. It can be proved by contradiction that $n$ is finite. The proof is identical to the one in $\mathrm{BC}$ in which the number of classes that make up the class partition for marriage is shown to be finite.

The implications of this theorem are as follows. First, singles can be split up into $n$ distinct classes. In equilibrium, matches, either cohabiting or marital, take place only between the two sexes in the same class. Second, the class partition for marriage is consistent with that of cohabitation. Third, the aggregate matching induced by class partitioning is not Pareto optimal due to imperfect information. That is, a low-type single (say, a man) can increase his welfare through mismatch in cohabitation (this can be achieved by missignalling at the partner's expense). A cohabiting individual of higher type (a woman) will suffer from a utility loss in comparison to what she could get when matched with a partner of the same class under perfect information, and she has to face the prospect of re-entering the marriage market and incurring more search costs after the relationship dissolves. The next section addresses the issue of risk in greater detail. 


\section{Risk premia, cohabitation and marriage}

This section addresses issues related to the risk of mis-match and the risk aversion of individuals. We do not however, allow strategic interaction among market participants. That is, we rule out the possibility that individuals deliberately mis-represent themselves. For simplicity, we drop the conditioning variable $\widetilde{m}_{e}$ in what follows. The results are obtained by linking the decision-maker's optimal policies in stages 2 and 3. We begin with the following proposition:

Proposition 2 An individual is more discriminating when deciding to marry than when deciding to cohabit. That is, for any single individual, the reservation pizazz level for a potential spouse is set higher than the reservation pizazz level for a potential cohabiting partner. This is solely because $G(x)$ is riskier than $F(m)$.

Proof: Consider a woman in the top-class. For this woman, $\widetilde{m}_{e} \in\left[m_{1}, \bar{m}\right]$ and $R\left(\widetilde{m}_{e}\right)$ $=\bar{R}$. If we write $m_{r}$ in place of $m_{1}$ and $x_{r}$ in place of $x_{1}$ to emphasize that these are reservation cut-off levels, we have $\varphi_{\tilde{m}}\left(m_{r}\right)=\bar{R}$ and $\psi\left(x_{r}\right)=\bar{R}$. From (4) we have,

$$
\bar{R}=\frac{m_{r}+\lambda E_{x \mid m=m_{r}} J(x)}{r+\lambda} .
$$

Using $\bar{R}=x_{r} / r$ and rearranging terms yields,

$$
\lambda\left[E_{x \mid m=m_{r}} J(x)-\bar{R}\right]=x_{r}-m_{r} .
$$

From Appendix (2) we have,

$$
x_{r}-m_{r}=\frac{\lambda}{r} \int_{x_{r}}^{\bar{x}}\left(x-x_{r}\right) d_{x} Q\left(x, m_{r}\right)>0 .
$$

where $d_{x} Q$ means differentiating $Q$ w.r.t. $x$. The above equation holds not just for the top-class, but for any class [Note 1]. Notice that (12) has two interpretations. The first is similar to the one found in the literature on the costs and benefits of searching for a job or for a marriage partner. The left hand side represents the opportunity cost of searching one more time with a marriage proposal or job offer of $x_{r}$ at hand. The right hand side represents the prospective benefit of this search, which is the expected discounted utility associated with a future possible draw of $x>x_{r}$. Because cohabitation unions precede marriage, individuals may miss out on the right husband or wife if they set reservation levels for cohabitation too high. Rational individuals should set lower reservation levels for cohabitation than for marriage because observed pizazz is a noisy signal of true pizazz. 
A second interpretation is based on a comparison of the distributions $F(m)$ and $G(x)$. Note that $F(m)$ second-order stochastically dominates $G(x)$. That is, $G(x)$ is riskier than $F(m)$. For example, in the normal distribution case, it can be shown that $G(x)$ is just a mean-preserving spread of $F(m)$ since $\mu_{m}=\mu_{x}$ and $\sigma_{m}^{2}<\sigma_{x}^{2}$. Recall that $G(x)$ is the distribution of true pizazz related to marriage search, while $F(m)$ is the distribution of expected pizazz underlying cohabitation search. Before a single individual receives any offers, there are only two available pieces of information that matter: the prior distributions of $G(x)$ and $F(m)$. Based on this, the individual at the pre-draw stage has to make an ex-ante decision on the optimal policy to be followed when cohabiting or marrying. Hence, his or her rational response to the higher risk of $G(x)$ than $F(m)$ is to become more selective in considering a long-run marital partnership over a potentially short-run cohabiting partnership. The term $R P \equiv x_{r}-m_{r}$ can therefore be interpreted as a kind of risk premium that compensates the single individual for the higher risk in marriage versus cohabitation.

Proposition 3 If one individual is in a higher class of cohabitation than another, she or he must be in a higher class of marriage as well. That is, for two people indexed by $k$ and $k+1, m_{r}^{k}>m_{r}^{k+1} \Rightarrow x_{r}^{k}>x_{r}^{k+1}$.

Proof: Combining the optimal search policies in stage 2 and 3 we have, $x_{r}=r R$ $=r \varphi\left(m_{r}\right)$. Using (7), we have $d x_{r} / d m_{r}=r \varphi^{\prime}\left(m_{r}\right)>0$. Therefore, an individual with higher reservation pizazz for cohabitation demands a higher reservation pizazz for marriage than an individual with lower reservation pizazz for cohabitation.

Proposition 4 If the degree of first-order stochastic dominance is large enough, the risk premium to compensate marriage risk is higher for an individual belonging to a higher cohabitation class than a lower cohabitation class. That is, for two people indexed $k$ and $k+1, m_{r}^{k}>m_{r}^{k+1} \Rightarrow x_{r}^{k}-m_{r}^{k}>x_{r}^{k+1}-m_{r}^{k+1}$.

Proof: This follows from

$$
R P^{\prime}\left(m_{r}\right) \geq 0 \Leftrightarrow \int_{x\left(m_{r}\right)}^{\bar{x}}\left[-\frac{\partial Q\left(x, m_{r}\right)}{\partial m_{r}}\right] d x \geq P\left(x \geq x_{r} \mid m=m_{r}\right) .
$$

The derivation of this result (see appendix (4)) uses (12) as defining an implicit function: $x_{r}=x\left(m_{r}\right)$. This proposition asserts that risk premia rise with the level of cohabitation reservation pizazz, provided that the probability of the cohabiting individual satisfying his or her partner's marital reservation demand is lower than the degree of the first-order stochastic dominance reflected by the integral of $-\partial Q / \partial m>0$. Therefore, an individual demanding a higher level for the cohabiting individual's pizazz requests a larger risk premium in establishing a marital partnership. 
From (12), we can see that the difference in $R P$ among classes originates from the difference in their $m_{r}$ through the underlying distribution, $Q\left(\cdot, m_{r}\right)$. According to this proposition, different classes may each have a different $R P$ under certain conditions since their cohabitation reservations $m_{r}$ are different. One can verify that in the normal distribution case, $R P^{\prime}\left(m_{r}\right)=0$. Under normality, the classes within which marriages occur are simply a uniform shift to the right of the classes within which cohabiting unions occur. They are shifted by a single amount equal to $R P\left(m_{r}\right)$ which is independent of $m_{r}$ and constant. This is summarized in the following corollary:

Corollary 1 The risk premium under the normality assumption is invariant across classes even if their $m_{r}$ 's are different.

Proposition 5 For any risk-neutral individual in any class, the risk premium needed to compensate for the higher risk of marriage over cohabitation is equal to the quantity of marital risk multiplied by its price.

Proof: Re-writing (12) to its equivalent (see Appendix (5)):

$$
x_{r}-m_{r}=P\left(x<x_{r} \mid m=m_{r}\right) E\left[\xi\left(x_{r}-x\right) \mid x<x_{r}, m=m_{r}\right]
$$

In the above, the quantity of risk [Note 2], $P\left(x<x_{r} \mid m=m_{r}\right)$, is the probability that the true pizazz of the cohabiting partner (say, a man) turns out to be below the individual (say, a woman)'s reservation level for marriage even though he was acceptable as a cohabiting partner. The 'price' of this mismatch risk is the expectation of $\xi\left(x_{r}-x\right)$, conditional on this disappointing revelation. The term $\xi=(1+r / \lambda)^{-1}<1$ is the modifying factor in the measurement of the risk price. The higher the discount rate $r$, and the lower the transition rate $\lambda$ of cohabitation to marriage, the smaller will be the effect of this multiplier on the risk price. The term, $\left(x_{r}-m_{r}\right)$, is the loss in utility compared with what the woman would have gained had she chosen a partner with $x>x_{r}$ as well as $m \geq m_{r}$ (that is, if the woman had chosen a man who was both a acceptable cohabiting partner and an acceptable husband).

In the normal distribution case, equation (14) reduces to an equation (see Appendix (6)), which implicitly defines the risk premium $R P$ as a function of $\sigma_{1}^{2}$, the variance of $(X \mid y)$ :

$$
x_{r}-m_{r}=\sigma_{1} \xi \phi\left(\frac{x_{r}-m_{r}}{\sigma_{1}}\right)\left[1-\xi \Phi\left(\frac{x_{r}-m_{r}}{\sigma_{1}}\right)\right]^{-1} .
$$

Recalling that $\sigma_{1}^{2}=w \sigma^{2}$ and $w^{\prime}\left(\sigma_{\varepsilon}^{2}\right)>0$, we see that if $\sigma_{\varepsilon}^{2}=0$ (that is, there is no noise), $R P$ would vanish so that the $\mathrm{BC}$ result is restored. Furthermore, one can derive from (15) that $\partial R P / \partial \sigma_{1}=R P / \sigma_{1}>0$. This implies that the smaller the value of $\sigma_{\varepsilon}^{2}$, the larger the overlap between the classes for cohabitation and marriage. Therefore, the following corollary results: 
Corollary 2 The risk premium RP under the normality assumption increases as the noise variance $\sigma_{\varepsilon}^{2}$ increases. As the variance $\sigma_{\varepsilon}^{2}$ increases, the overlap between the class partition for marriage and cohabitation decreases.

So far, individuals are assumed to be risk neutral. We now relax this assumption. Note however, that relaxing this assumption does not alter the results obtained in the last section.

Proposition 6: For any risk-averse individual in any class, the total risk premium can be decomposed into two parts. One part compensates the individual for bearing the risk due to the second-order stochastic dominance of $F(m)$ over $G(x)$; the other part is due to the individual's risk aversion.

Proof: We assume that the individual's utility function $u(x)$ possesses the usual properties of being strictly increasing and concave. Accordingly, (12) can be changed to

$$
u\left(x_{r}\right)-u\left(m_{r}\right)=\frac{\lambda}{r} \int_{x_{r}}^{\bar{x}}\left[u(x)-u\left(x_{r}\right)\right] d_{x} Q\left(x, m_{r}\right)>0 .
$$

The application of Jensen's inequality to the above equation yields,

$$
u\left(x_{r}\right)-u\left(m_{r}\right)<\xi P\left(x<x_{r} \mid m=m_{r}\right) E\left[\left(u\left(x_{r}\right)-u(x)\right) \mid x<x_{r}, m=m_{r}\right] .
$$

We can consider $x$ to be $m_{r}$ plus an actuarially neutral gamble $z\left(=x-m_{r}\right)$ in the sense that $E_{x \mid m_{r}}(z)=0$. Note that $\sigma_{x \mid m_{r}}^{2}(z)=\sigma_{1}^{2}$. Taking the second-order Taylor series expansion of $u(x)$ around $m_{r}$, the first-order expansion of $u\left(x_{r}\right)$ around $m_{r}$, and using the Pratt-Arrow measure of local risk premium (see chapter 4 of Copeland and Weston (1979) for a definition), yields (see Appendix (7)),

$$
R P_{p a}=-\frac{\sigma_{1}^{2}}{2} \frac{u^{\prime \prime}\left(m_{r}\right)}{u^{\prime}\left(m_{r}\right)}>0 .
$$

In the above, $R P_{p a}$ captures the difference between the quantities on both sides of Jensen's inequality or the degree of the agent's risk aversion if the risk in question is not too large. Mathematical manipulation yields,

$x_{r}-m_{r}+\xi R P_{p a}=P\left(x<x_{r} \mid m=m_{r}\right) E\left[\xi\left(x_{r}-x\right) \mid x<x_{r}, m=m_{r}\right]+\delta \xi R P_{p a}$,

where

$$
\delta=\frac{1}{\sigma_{1}^{2}} \int_{\underline{x}}^{x_{r}}\left(x-m_{r}\right)^{2} d_{x} Q\left(x, m_{r}\right)<1 .
$$


Thus

$$
x_{r}-m_{r}+(1-\delta) \xi R P_{p a}=P\left(x<x_{r} \mid m=m_{r}\right) E\left[\xi\left(x_{r}-x\right) \mid x<x_{r}, m=m_{r}\right],
$$

which reduces to (14) if $u^{\prime \prime}=0$. This result concerning the decomposition of the risk premium depends however, on the assumption that $\sigma_{\varepsilon}^{2}$ is small so that the matching risk is not very large, and the Arrow-Pratt approximation can therefore be used.

From (18), it appears that risk aversion only matters when dividing the total risk premium into two parts for compensation: $\left(x_{r}-m_{r}\right)$ compensates the searching single individual for signalling risks; $(1-\delta) \xi R P_{p a}$ compensates for risk aversion. However, this may not be the case even when the underlying distributions are normal. In this case under the optimality condition (equation 18), one can find (see Appendix (8)) that $\left(x_{r}-m_{r}\right)$ is lower under risk aversion than under risk neutrality, and risk aversion may make a difference in affecting the total risk premium in addition to the decomposition.

Corollary 3 The total risk premium is lower under risk aversion than under risk neutrality in the normal distribution case.

In this case, singles will not set their marriage reservation pizazz $x_{r}$ too high given their $m_{r}$. This is similar to the situation in which they do not set $m_{r}$ too high given $x_{r}$, in order not to miss out on the right husband or wife. This is because they are now more concerned than in the risk-neutrality case about the positive discount rate, difficulties in revealing each other's true type, and uncertainty about receiving offers of cohabitation and marriage.

\section{Conclusion}

Marriages today are commonly preceded by a period of cohabitation. For many couples, this period of cohabitation serves as a trial marriage. It is a period in which they can decide if their cohabiting partner is the right choice as a spouse.

Despite a vast empirical literature, mainly in demography, there are few theoretical models that can be used to study the modern phenomenon of cohabitation followed by marriage. This paper develops such a model using the framework of a two-sided searchmatching model. To this framework, the paper adds imperfect information, learning and risk. This extended model is consistent with empirical findings from demographic studies on cohabitation and marriage.

\section{Acknowledgments}

We thank seminar participants at the University of Toronto for useful comments. 


\section{Appendices}

\section{(1) The joint distribution of observed and true pizazz.}

Suppose that the joint density of true pizazz, $X$ and observed pizazz, $Y$ is denoted $f_{X, Y}(x, y)$. The distribution of true pizazz conditional on observed pizazz is

$$
Q_{X \mid Y}(x \mid y)=\operatorname{Pr}(X \leq x \mid y)=\frac{\int_{\underline{x}}^{x} f_{X, Y}(x, y) d x}{\int_{\underline{x}}^{\bar{x}} f_{X, Y}(x, y) d x} .
$$

The expectation of true pizazz conditional on observed pizazz, denoted $m(y)$ can be written as

$$
m(y)=E(X \mid Y=y)=\int_{\underline{x}}^{\bar{x}} x q_{X \mid Y}(x \mid y) d x=\frac{\int_{\underline{x}}^{\bar{x}} x f_{X, Y}(x, y) d x}{\int_{\underline{x}}^{\bar{x}} f_{X, Y}(x, y) d x} .
$$

The distribution of the random variable $M=E(X \mid Y)$ is given by

$$
F(m)=\operatorname{Pr}(M \leq m)=\operatorname{Pr}[Y \leq y(m)]=\int_{\underline{y}}^{y(m)} \int_{\underline{x}}^{\bar{x}} f_{X, Y}(x, y) d x d y .
$$

(2) Derivation of (5).

Expanding the expectation of the value function in stage 3, we obtain,

$$
E_{x \mid m} J=E_{x \mid m} \max (\psi, R)=\operatorname{Pr}(\psi \geq R) E_{x \mid m}(\psi \mid \psi \geq R)+\operatorname{Pr}(\psi<R) R .
$$

Using $x_{r}=r R\left(\widetilde{m}_{e}\right)$, this may be simplified as follows:

$$
\begin{aligned}
E J & =\frac{1}{r}\left\{\operatorname{Pr}\left(x \geq x_{r} \mid y\right)\left[E\left(x \mid x \geq x_{r}\right)\right]+\operatorname{Pr}\left(x<x_{r} \mid y\right) x_{r}\right\} \\
& =\frac{1}{r}\left\{\operatorname{Pr}\left(x \geq x_{r} \mid y\right)\left[E\left(x \mid x \geq x_{r}\right)-x_{r}\right]+x_{r}\right\} .
\end{aligned}
$$

Writing $\int_{x_{r}}^{\bar{x}} d_{x} Q(x, m)$ in place of $\operatorname{Pr}\left(x \geq x_{r} \mid y\right)$ and using $x_{r}=r R\left(\widetilde{m}_{e}\right)$ yields,

$$
E J=\frac{1}{r} \int_{x_{r}}^{\bar{x}}\left(x-x_{r}\right) d Q(x, m)+R\left(\widetilde{m}_{e}\right)
$$


The integral term can be changed to $-\int_{x_{r}}^{\bar{x}}\left(x-x_{r}\right) d_{x}\left[1-Q_{x}(x, m)\right]$. Applying the technique of integration by parts and using $\psi_{\widetilde{m}_{e}}\left(x_{r}\right)=R\left(\widetilde{m}_{e}\right)$ yields (5) in the text of the paper.

\section{(3) Derivation of (8).}

We begin by re-writing (2):

$$
\begin{aligned}
{\left[\alpha\left(\widetilde{m}_{e}\right)+r\right] R\left(\widetilde{m}_{e}\right) } & =\alpha\left(\widetilde{m}_{e}\right) E_{m} \max \left\{\varphi_{\widetilde{m}_{e}}(m), R\left(\widetilde{m}_{e}\right)\right\} \\
& =\alpha\left(\widetilde{m}_{e}\right)\left\{\operatorname{Pr}(\varphi \geq R) E\left[\varphi_{\tilde{m}_{e}}(m) \mid \varphi \geq R\right]+\operatorname{Pr}(\varphi<R) R\left(\widetilde{m}_{e}\right)\right\}
\end{aligned}
$$

Re-arranging terms yields,

$$
r R\left(\widetilde{m}_{e}\right)=\alpha\left(\widetilde{m}_{e}\right) \operatorname{Pr}(\varphi \geq R)\left\{E\left[\varphi_{\widetilde{m}_{e}}(m) \mid \varphi \geq R\right]-R\left(\widetilde{m}_{e}\right)\right\} .
$$

Using $\varphi_{\tilde{m}_{e}}\left(m_{r}\right)=R\left(\widetilde{m}_{e}\right)$, the above equation can be re-written as

$$
\begin{aligned}
R\left(\widetilde{m}_{e}\right) & =\frac{\alpha\left(\widetilde{m}_{e}\right)}{r} \int_{m_{r}}^{\bar{m}}\left[\varphi_{\tilde{m}_{e}}(m)-\varphi_{\widetilde{m}_{e}}\left(m_{r}\right)\right] d F\left(m \mid \widetilde{m}_{e}\right) \\
& =-\frac{\alpha\left(\widetilde{m}_{e}\right)}{r} \int_{m_{r}}^{\bar{m}}\left[\varphi_{\widetilde{m}_{e}}(m)-\varphi_{\widetilde{m}_{e}}\left(m_{r}\right)\right] d\left[1-F\left(m \mid \widetilde{m}_{e}\right)\right] .
\end{aligned}
$$

Integrating by parts and noting that $F\left(\bar{m} \mid \widetilde{m}_{e}\right)=1$ yields,

$$
R\left(\widetilde{m}_{e}\right)=\frac{\alpha\left(\widetilde{m}_{e}\right)}{r} \int_{m_{r}}^{\bar{m}}\left[1-F\left(m \mid \widetilde{m}_{e}\right)\right] \varphi_{\widetilde{m}_{e}}^{\prime}(m) d m .
$$

Substituting (7) and (6) in the above yields (8) in the text of the paper.

(4) Derivation of (13).

In the interpolation of equation (12), we think of $x_{r}$ and $m_{r}$ as having a relationship such as $x_{r}=x\left(m_{r}\right)$ as if they were continuous variables. Differentiating this equation w.r.t. $m_{r}$ yields,

$$
x^{\prime}\left(m_{r}\right)=\left\{1+\frac{\lambda}{r} \int_{x_{r}}^{\bar{x}}\left[-\frac{\partial Q\left(x, m_{r}\right)}{\partial m_{r}}\right] d x\right\}\left\{1+\frac{\lambda}{r}\left[1-Q\left(x_{r}, m_{r}\right)\right]\right\}^{-1}>0
$$


We know that $R P^{\prime}\left(m_{r}\right)>0$ iff $x^{\prime}\left(m_{r}\right)>1$. From the above, it follows that

$$
\int_{x_{r}}^{\bar{x}}\left[-\frac{\partial Q\left(x, m_{r}\right)}{\partial m_{r}}\right] d x>1-Q\left(x_{r}, m_{r}\right),
$$

From the above we obtain (13).

(5) Derivation of (14).

We change (12) in the following manner:

$$
\begin{aligned}
x_{r}-m_{r} & =\frac{\lambda}{r}\left(\int_{\underline{x}}^{\bar{x}}-\int_{\underline{x}}^{x_{r}}\right)\left(x-x_{r}\right) d_{x} Q\left(x, m_{r}\right) \\
& =\frac{\lambda}{r}\left(m_{r}-x_{r}\right)+\frac{\lambda}{r} \int_{\underline{x}}^{x_{r}}\left(x_{r}-x\right) d_{x} Q\left(x, m_{r}\right)
\end{aligned}
$$

Using the conditional expectation definition and simplifying the expression gives (14) in the paper.

(6) Derivation of (15).

Using formula

$$
E\left(x \mid x>x_{0}\right)=\mu+\sigma \phi\left(\frac{\mu-x_{0}}{\sigma}\right) \Phi^{-1}\left(\frac{\mu-x_{0}}{\sigma}\right), \text { for } x \sim N\left(\mu, \sigma^{2}\right),
$$

we transform (12) into

$$
\begin{aligned}
x_{r}-m_{r} & =\frac{\lambda}{r} \Phi\left(\frac{m_{r}-x_{r}}{\sigma_{1}}\right)\left[E\left(x \mid x>x_{r}, m_{r}\right)-x_{r}\right] \\
& =\frac{\lambda}{r} \Phi\left(\frac{m_{r}-x_{r}}{\sigma_{1}}\right)\left[\sigma_{1} \phi\left(\frac{m_{r}-x_{r}}{\sigma_{1}}\right) \Phi^{-1}\left(\frac{m_{r}-x_{r}}{\sigma_{1}}\right)-\left(x_{r}-m_{r}\right)\right] \\
& =\frac{\lambda}{r}\left[\sigma_{1} \phi\left(\frac{m_{r}-x_{r}}{\sigma_{1}}\right)-\left(x_{r}-m_{r}\right) \Phi\left(\frac{m_{r}-x_{r}}{\sigma_{1}}\right)\right] .
\end{aligned}
$$

Collecting terms, noting that $1-\Phi(z)=\Phi(-z)$ and $\phi(-z)=\phi(z)$, and using notation $\xi$, yields (15).

\section{(7) Derivation of (18).}


Changing (16) in the same way as in (19) gives the following:

$$
\left(1+\frac{\lambda}{r}\right) u\left(x_{r}\right)-u\left(m_{r}\right)-\frac{\lambda}{r} E\left[u(x) \mid m_{r}\right]=\frac{\lambda}{r} \int_{\underline{x}}^{x_{r}}\left[u\left(x_{r}\right)-u(x)\right] d Q
$$

Taking the first-order Taylor expansion of $u\left(x_{r}\right)$ around $u\left(m_{r}\right)$ and the second-order Taylor expansion of $u(x)$ around $u\left(m_{r}\right)$ gives

$$
\begin{aligned}
& u\left(x_{r}\right) \cong u\left(m_{r}\right)+u^{\prime}\left(m_{r}\right)\left(x_{r}-m_{r}\right) \\
& u(x) \cong u\left(m_{r}\right)+u^{\prime}\left(m_{r}\right)\left(x-m_{r}\right)+\frac{u^{\prime \prime}\left(m_{r}\right)}{2}\left(x-m_{r}\right)^{2}
\end{aligned}
$$

Note that $z=x-m_{r}$, and $E\left(z \mid m_{r}\right)=0$. We know from (22) that

$$
E\left[u(x) \mid m_{r}\right] \cong u\left(m_{r}\right)+\frac{u^{\prime \prime}\left(m_{r}\right)}{2} \sigma_{1}^{2}
$$

Substituting from (21) and (23) into (20) and recalling the definition of $\xi$ and $R P_{p a}$ yields

$$
x_{r}-m_{r}+\xi R P_{p a} \cong \xi \int_{\underline{x}}^{x_{r}}\left[\left(x_{r}-x\right)-\frac{u^{\prime \prime}\left(m_{r}\right)}{2 u^{\prime}\left(m_{r}\right)}\left(x-m_{r}\right)^{2}\right] d Q
$$

which can easily be changed to (17) using the definition of $R P_{p a}, \delta$ and conditional expectation operator.

(8) Geometric illustration of difference in $x_{r}-m_{r}$ (denoted $\tau$ ).

In the normal distribution case, (18) takes the form of

$$
x_{r}-m_{r}+\triangle=\xi \sigma_{1} \phi\left(\frac{x_{r}-m_{r}}{\sigma_{1}}\right)\left[1-\xi \Phi\left(\frac{x_{r}-m_{r}}{\sigma_{1}}\right)\right]^{-1}
$$

where $\triangle=(1-\delta) \xi R P_{p a}\left[1-\xi \Phi\left(\frac{x_{r}-m_{r}}{\sigma_{1}}\right)\right]^{-1}$. Denote by $g(\tau)$ the function (total risk premium) on the $R H S$ of either (15) or (24). Then, $g^{\prime}(\tau)=g(g-\tau) / \sigma_{1}^{2}$ and $\sigma_{1}^{2} g^{\prime \prime}(\tau)=g^{\prime}(\tau)(2 g-\tau)-g$. Denote by $\tau^{r n}$ and $\tau^{r a}$ the risk-neutral and risk-averse solutions to equations (15) and (24), respectively. We see that $g^{\prime}\left(\tau^{r n}\right)=0, g^{\prime}\left(\tau^{r a}\right)$ $>0$, and $g^{\prime \prime}\left(\tau^{r n}\right)=-g\left(\tau^{r n}\right)<0$. Therefore, in the first quadrant of $(\tau, g)$-space, $g$ $=g(\tau)$ traces a curve that is concave and flat at $\tau^{r n}$, but upward-sloped at $\tau^{r a}$. Denote by $h(\tau)$ the function on the $L H S$ of (24). The term $h(\tau)$ represents a curve, which lies everywhere above $45^{0}$ line that is just the $L H S$ of (15). The two points that result from intersection among this line and curves show that $\tau^{r a}<\tau^{r n}$ and $g\left(\tau^{r a}\right)<g\left(\tau^{r n}\right)$. The last inequality, however, might be reversed for distributions that are not normal. 


\section{Notes}

1. The general relation related to (12) for class $i$ or $\widetilde{m}_{e} \in\left(m_{i}, m_{i-1}\right]$ should take the form of

$$
x_{i}-m_{i}=\frac{\lambda}{r} \int_{x_{i}}^{x_{i}-1}\left(x-x_{i}\right) d_{x} Q\left(x, m_{i}\right)
$$

2. Stringently, the quantity of risk for class $i$ should be measured as

$$
\begin{aligned}
P(x & \left.<x_{i} \mid m_{i} \leq m<m_{i-1}\right)=\int_{-\infty}^{x_{i}} f\left(x \mid y_{i} \leq y<y_{i-1}\right) d x \\
& =\int_{-\infty}^{x_{i}} \int_{y_{i}}^{y_{i-1}} f(x, y) d y d x / \int_{y_{i}}^{y_{i}-1} f_{Y}(y) d y
\end{aligned}
$$

using $y^{\prime}(m)>0$. The price of risk for this class is

$$
\begin{aligned}
& E\left(x_{i}-x \mid x<x_{i}, m_{i} \leq m<m_{i-1}\right) \\
&=\int_{-\infty}^{x_{i}} \int_{y_{i}}^{y_{i-1}}\left(x_{i}-x\right) f(x, y) d x d y / \int_{-\infty}^{\infty} \int_{y_{i}}^{y_{i-1}} f(x, y) d x d y .
\end{aligned}
$$

However, less stringently, one can still condition on $m=m_{r}$ in (14) to address the issue of mismatch risk. This is the risk that the current partner is only good enough as a cohabitor since $m=m_{r}$ but not good enough to be a spouse since $x<x_{r}$. 


\section{References}

Bloch, F. and H. Ryder (2000), "Two-Sided Search, Marriages and Matchmakers." International Economic Review, February, 41(1), 93-115.

Bumpass, L. and J. A. Sweet (1989), "National Estimates of Cohabitation.” Demography, $26,615-625$.

Bumpass, L., J. A. Sweet and A. Cherlin (1991), "The role of cohabitation in declining rates of marriage." Journal of Marriage and the Family, 53(4), 913-927.

Burdett, K. and M. Coles (1997), "Marriage and Class." The Quarterly Journal of Economics, CXII, February, 141-168.

Burdett, K. and M. Coles (1999), "Long-term Partnership Formation : Marriage and Employment.” The Economic Journal, 109, June, 307-334.

Chade, H. (1999) Two-Sided Search, Heterogeneity, and Noisy Observable Types, mimeo, Arizona State University.

Copeland, E.T. and J.F. Weston (1983), Financial Theory and Corporate Policy, second edition, Addison-Wesley Publishing Company.

Ermisch, J. and M. Francesconi (2000), "Cohabitation in Great Britain: Not for Long, but Here to Stay.” Journal of the Royal Statistical Society, Series A, 163(2), 153-71.

Kiernan, K. E. (1996), "Partnership Behavior in Europe: Recent Trends and Issues." in Europe's Population in the 1990s edited by D. Coleman. Oxford: Oxford University Press.

Rao Sahib P. and X. Gu (2000), "Living in Sin" and Marriage: a Matching Model, Journal of Population Economics, forthcoming.

Sargent, T. J. (1987), Dynamic Macroeconomic Theory, Harvard University Press.

Shimer R. and L. Smith (2000), Assortative Matching and Search 68(2), Econometrica, March, 343-69. 
Demographic Research - Volume 6, Article 13

Waters, M. S. and R. Ressler (1999), "An economic model of cohabitation and divorce" Journal of Economic Behavior and Organization, 40, 195-206. 
Demographic Research - Volume 6, Article 13 